\title{
Apoptosis turns 21
}

\author{
Arjan W. Griffioen ${ }^{1}$ Patrycja Nowak-Sliwinska ${ }^{2}$
}

Published online: 13 November 2017

(C) Springer Science+Business Media, LLC, part of Springer Nature 2017

2016 saw Apoptosis come of age, celebrating its 21 st anniversary. Over the past 21 years, apoptosis has become a heavily studied process of cell death. While the discovery and description dates back to the mid-nineteenth century, renewed interest in programmed cell death started in the 1960s and the term 'apoptosis' was attributed to this process in 1972 [1]. It is now fully clear that apoptosis is a common process of cell death, present in multicellular organisms, even those present in the kingdoms of plants, fungi and bacteria.

Apoptosis serves the scientific community through the publication of high quality research in the form of original articles, reviews, short communications and commentaries. In addition, it is the journal's intention to provide a platform for scientific discussion and debate. The anniversary of the journal coincided with a change in Editorship and we would again like to take this opportunity to thank Dr. Mels Sluyser for his stewardship.

As the new editors, we are looking at ways to continue to develop the journal, to provide a timely and efficient peer review process, and to increase the visibility of its content. For example, Springer Nature has recently launched a content-sharing intitiative, SharedIt, which enables researchers to share research with colleagues and general audiences. In

Arjan W. Griffioen

aw.griffioen@vumc.nl

Patrycja Nowak-Sliwinska

patrycja.nowak-sliwinska@unige.ch

1 Angiogenesis Laboratory, Department of Medical Oncology, VU University Medical Center, 1081 HV Amsterdam, The Netherlands

2 School of Pharmaceutical Sciences, University of Geneva, Geneva, Switzerland addition, we have recently restructured the editorial board which now comprises an enthusiastic crew of internationally renowned experts to help us select and review the most noteworthy papers in all aspects of apoptosis research.

Several excellent papers have been published in Apoptosis over the last several years. Cardioprotective effects were reported in ischemia reperfusion injury by salidroside through inhibition of apoptosis in myocardial cells and inhibition of pro-inflammatory cytokines [2], and by berberine, though attenuation of Notch1/Hes1-PTEN/Akt signalling [3]. The latter compound also was found to prevent the nitric oxide-induced apoptosis in chondrocytes, resulting in decreased cartilage degeneration and amelioration of osteoarthritis [4]. Zhang et al. reported on the inhibitor INK128 suppressing the growth of neuroblastoma in in vitro and in vivo models, enhancing the effect of doxorubicin at inducing apoptosis [5]. Another highly cited paper was on the protective ability of phosphocreatine on endothelial cells, through the modulation of PI3K/Akt/eNOS pathway, a process that prevents the endothelial dysfunction in atherosclerosis [6]. Apoptosis also published a series of excellent reviews on Bcl-2 targeted drug discovery [7], the biology of Toll/Interleukin-1 receptor superfamily of proteins [8, 9], apoptosis in mammalian oocytes [10] and plant-derived anticancer drugs [11].

As the journal has now entered adulthood, we will be changing the journal's cover design and we encourage all authors to submit their microscopy images related to apoptotic cell death, (deadline 1st March 2018). A selection of these will then be published in the journal, while the most striking image will form the journal cover for the coming year.

Another new feature, starting January 2018, is the Editors' Choice section, which will highlight and discuss the most noteworthy recently published articles. 
We are confident that Apoptosis will succeed in becoming a stronger and better recognized high quality journal for publication of research on the regulation of cell death.

\section{References}

1. Kerr JF, Wyllie AH, Currie AR (1972) Apoptosis: a basic biological phenomenon with wide-ranging implications in tissue kinetics. Br J Cancer 26:239-257

2. Zhu L, Wei T, Gao J, Chang X, He H, Luo F et al (2015) The cardioprotective effect of salidroside against myocardial ischemia reperfusion injury in rats by inhibiting apoptosis and inflammation. Apoptosis 20:1433-1443

3. Yu L, Li F, Zhao G, Yang Y, Jin Z, Zhai M et al (2015) Protective effect of berberine against myocardial ischemia reperfusion injury: role of Notch1/Hes1-PTEN/Akt signaling. Apoptosis 20:796-810

4. Zhou Y, Liu SQ, Yu L, He B, Wu SH, Zhao Q et al (2015) Berberine prevents nitric oxide-induced rat chondrocyte apoptosis and cartilage degeneration in a rat osteoarthritis model via AMPK and p38 MAPK signaling. Apoptosis 20:1187-1199
5. Zhang H, Dou J, Yu Y, Zhao Y, Fan Y, Cheng J et al (2015) mTOR ATP-competitive inhibitor INK128 inhibits neuroblastoma growth via blocking mTORC signaling. Apoptosis 20:50-62

6. Ahsan A, Han G, Pan J, Liu S, Padhiar AA, Chu P et al (2015) Phosphocreatine protects endothelial cells from oxidized lowdensity lipoprotein-induced apoptosis by modulating the PI3K/ Akt/eNOS pathway. Apoptosis 20:1563-1576

7. Kvansakul M, Hinds MG (2015) The Bcl-2 family: structures, interactions and targets for drug discovery. Apoptosis 20:136-150

8. Narayanan KB, Park HH (2015) Toll/interleukin-1 receptor (TIR) domain-mediated cellular signaling pathways. Apoptosis 20:196-209

9. Ve T, Williams SJ, Kobe B (2015) Structure and function of Toll/ interleukin-1 receptor/resistance protein (TIR) domains. Apoptosis 20:250-261

10. Tiwari M, Prasad S, Tripathi A, Pandey AN, Ali I, Singh AK et al (2015) Apoptosis in mammalian oocytes: a review. Apoptosis 20:1019-1025

11. Gali-Muhtasib H, Hmadi R, Kareh M, Tohme R, Darwiche N (2015) Cell death mechanisms of plant-derived anticancer drugs: beyond apoptosis. Apoptosis 20:1531-1562 